\title{
LAMBERT SUMMABILITY OF ORTHOGONAL SERIES
}

\section{RICHARD BELLMAN}

If we define Lambert summability of a series, $\sum_{1}^{\infty} a_{n}$, in terms of the existence of the limit

$$
L\left(a_{n}\right)=\lim _{x \rightarrow 1-0}(1-x) \sum_{1}^{\infty} \frac{n a_{n} x^{n}}{1-x^{n}}
$$

we have, by a well known theorem of Hardy-Littlewood [1],1 that $C\left(a_{n}\right) \rightarrow L\left(a_{n}\right) \rightarrow A\left(a_{n}\right) ; C\left(a_{n}\right), A\left(a_{n}\right)$ are respectively the Cesàro and Abel means of the series $\sum_{1}^{\infty} a_{n}$.

The proof of $C\left(a_{n}\right) \rightarrow L\left(a_{n}\right)$ is elementary in nature, but the proof of $L\left(a_{n}\right) \rightarrow A\left(a_{n}\right)$ requires the prime number theorem, and conversely the theorem $L\left(a_{n}\right) \rightarrow A\left(a_{n}\right)$ implies the prime number theorem.

For that reason, it is perhaps interesting to show that for orthogonal series of functions $f(x)$, belonging to $L^{2}$, the inclusion of $L\left(a_{n}\right)$ between $C\left(a_{n}\right)$ and $A\left(a_{n}\right)$ follows in completely elementary fashion.

That $C\left(a_{n}\right) \sim A\left(a_{n}\right)$ for orthogonal series of $L^{2}$ is a known result of Kaczmarz [2]. Hence it is sufficient to show that $L\left(a_{n}\right) \rightarrow C\left(a_{n}\right)$. In addition, it is further known that $C\left(a_{n}\right)$ is equivalent to the convergence of the partial sums of the orthogonal series $S_{2^{n}}(\theta)=\sum_{1}^{2^{n}} a_{k} \phi_{k}(\theta)$ [3] Therefore, finally, it comes to showing that Lambert summability implies the convergence of the partial sums $s_{2^{n}}(\theta)$, in order to prove the theorem.

Let $f(\theta) \subset L^{2}(a, b), a_{n}=\int_{a}^{b} f(\theta) \phi_{n}(\theta) d \theta$; where $\left(\phi_{n}(\theta)\right)$ is an orthonormal sequence in $(a, b), s_{n}(\theta)=\sum_{1}^{n} a_{n} \phi_{n}(\theta)$.

Write, where $x$ is $1-1 / 2^{n}$,

$$
U_{n}(\theta)=\sum_{1}^{\infty} k a_{k} \phi_{k}(\theta) \frac{(1-x) x^{k}}{1-x^{k}}-s_{2^{n}}(\theta)=T_{n}(\theta)+V_{n}(\theta)
$$

where

$$
\begin{gathered}
T_{n}(\theta)=\sum_{1}^{2^{n}} a_{k} \phi_{k}(\theta)\left(\frac{k(1-x) x^{k}}{1-x^{k}}-1\right), \\
V_{n}(\theta)=\sum_{2^{n}+1}^{\infty} k a_{k} \phi_{k}(\theta) \frac{(1-x) x^{k}}{1-x^{k}} .
\end{gathered}
$$

If $\lim _{n \rightarrow \infty} U_{n}(\theta)=0$, the result is proven. To that end, consider the

Received by the editors June 10, 1943.

1 Numbers in brackets refer to the references listed at the end of the paper. 
series

$$
\sum_{1}^{\infty}\left[U_{n}(\theta)\right]^{2}
$$

To prove convergence almost everywhere in $\theta$, it is sufficient to show

$$
\sum_{n=1}^{\infty} \int_{a}^{b}\left[U_{n}(\theta)\right]^{2} d \theta<\infty
$$

We have

$$
\sum_{n} \int_{a}^{b}\left[U_{n}(\theta)\right]^{2} d \theta \leqq 2 \sum_{n} \int_{a}^{b}\left[T_{n}(\theta)\right]^{2} d \theta+2 \sum_{n} \int_{a}^{b}\left[V_{n}(\theta)\right]^{2} d \theta
$$

Let us consider the convergence of each series separately.

$$
\begin{aligned}
\sum_{n} \int_{a}^{b}\left[T_{n}(\theta)\right]^{2} d \theta & =\sum_{n} \int_{a}^{b}\left(\sum_{1}^{2^{n}} a_{k} \phi_{k}(\theta)\left(\frac{k(1-x) x^{k}}{1-x^{k}}-1\right)\right)^{2} d \theta \\
& =\sum_{n}\left(\sum_{1}^{2^{n}} a_{k}^{2}\left(\frac{k(1-x) x^{k}}{1-x^{k}}-1\right)^{2}\right)
\end{aligned}
$$

where the $x$ appearing in $\sum_{1}^{2^{n}}$ is $1-1 / 2^{n}, n \geqq 1$.

Now

so that

$$
\begin{array}{ll}
1-x^{k} \leqq k(1-x), & 0 \leqq x \leqq 1, \\
1-x^{k} \geqq 1-\frac{k(1-x) x^{k}}{1-x^{k}} \geqq 0, &
\end{array}
$$

$$
\begin{aligned}
\sum_{n} \int_{a}^{b}\left[T_{n}(\theta)\right]^{2} d \theta & \leqq \sum_{n} \sum_{1}^{2^{n}} a_{k}^{2}\left(1-x^{k}\right)^{2} \leqq \sum_{n} \sum_{1}^{2^{n}} k^{2} a_{k}^{2}(1-x)^{2} \\
& \leqq \sum_{n} \frac{1}{2^{2 n}} \sum_{1}^{2^{n}} k^{2} a_{k}^{2} \leqq \sum_{k} a_{k}^{2} k^{2} \sum_{n \geqq \log _{2} k} 2^{-2 n} \leqq A \sum_{k} a_{k}^{2}
\end{aligned}
$$

and $\sum_{k} a_{k}^{2}<\infty$ since $f(x) \subset L^{2}(a, b)$.

Now for the second series $\sum_{n} \int_{a}^{b}\left[V_{n}(\theta)\right]^{2} d \theta$ :

$$
\begin{aligned}
\sum_{n} \int_{a}^{b}\left[V_{n}(\theta)\right]^{2} d \theta & =\sum_{n} \int_{a}^{b}\left(\sum_{2^{n}+1}^{\infty} a_{k} \phi_{k}(\theta) \frac{k(1-x) x^{k}}{1-x^{k}}\right)^{2} d \theta \\
& =\sum_{n}\left\{\sum_{2^{n}+1}^{\infty} k^{2} a_{k}^{2} \frac{(1-x)^{2} x^{2 k}}{\left(1-x^{k}\right)^{2}}\right\}
\end{aligned}
$$


where the $x$ appearing in $\sum_{2^{n+1}}^{\infty}$ is $1-1 / 2^{n}, n \geqq 1$.

Since $\left(1-2^{-n}\right)^{k}$ is a decreasing function of $k$,

$$
\begin{aligned}
\sum_{n} \sum_{2^{n}+1}^{\infty} k^{2} a_{k}^{2} & \frac{(1-x)^{2} x^{2 k}}{\left(1-x^{k}\right)^{2}} \\
& \leqq \sum_{n} \frac{1}{1-\left(1-2^{-n}\right)^{2^{n}}} \sum_{2^{n}+1}^{\infty} k^{2} a_{k}^{2}(1-x)^{2} x^{2 k} \\
& \leqq A \sum_{n} \sum_{2^{n}+1} k^{2} a_{k}^{2}(1-x)^{2} x^{2 k} .
\end{aligned}
$$

We can majorize $k^{2} \sum_{1}^{\infty} 2^{-2 n}\left(1-2^{-n}\right)^{2 k}$ by the integral

$$
\begin{aligned}
k^{2} \int_{0}^{\infty} 2^{-2 x}\left(1-2^{-x-1}\right)^{2 k} d x & =4 k^{2} \int_{1}^{\infty} 2^{-2 x}\left(1-2^{-x}\right)^{k} d x \\
& <4 k^{2} \int_{0}^{\infty} 2^{-2 x}\left(1-2^{-x}\right)^{2 k} d x \\
& =A k^{2} /(2 k+1)(2 k+2)
\end{aligned}
$$

which is obviously bounded.

Therefore we have proven the convergence of the series, which implies that $\lim _{n \rightarrow \infty} U_{n}(\theta)=0$ almost everywhere in $\theta$, which implies that

$$
L\left(a_{n}\right)=\lim _{n \rightarrow \infty} s_{2^{n}}(\theta)
$$

almost everywhere in $\theta$.

This is equivalent to what we set out to prove.

\section{REFERENCES}

1. Hardy and Littlewood, On a Tauberian theorem for Lambert's series, Proc. Lond. Math. Soc. (2) vol. 19 (1921) pp. 21-29. 583.

2. Kaczmarz and Steinhaus, Theorie der Orthogonalreihen, Warsaw, 1935, Theorem

3. Ibid., Theorem 585.

UNIVERSITY OF Wisconsin 